\title{
Precipitation of hydrides in high purity niobium after different treatments
}

\author{
F. Barkov, ${ }^{1}$ A. Romanenko, ${ }^{1,}$ a) Y. Trenikhina, ${ }^{1,2}$ and A. Grassellino ${ }^{1}$ \\ 1) Fermi National Accelerator Laboratory, Batavia, IL 60510, USA \\ ${ }^{2)}$ Physics Department, Illinois Institute of Technology, Chicago, IL 60616, USA
}

(Dated: 4 March 2014)

Precipitation of lossy non-superconducting niobium hydrides represents a known problem for high purity niobium in superconducting applications. Using cryogenic optical and laser confocal scanning microscopy we have directly observed surface precipitation and evolution of niobium hydrides in samples after different treatments used for superconducting RF cavities for particle acceleration. Precipitation is shown to occur throughout the sample volume, and the growth of hydrides is well described by the fast diffusion-controlled process in which almost all hydrogen is precipitated at $T=140 \mathrm{~K}$ within $\sim 30$ min. $120^{\circ} \mathrm{C}$ baking and mechanical deformation are found to affect hydride precipitation through their influence on the number of nucleation and trapping centers.

Keywords: superconducting RF, hydroden Q-disease, niobium

\section{INTRODUCTION}

Niobium can easily absorb hydrogen, which, depending on the concentration and temperature, may either stay as an interstitial or precipitate into ordered niobium hydride phases. In both cases superconducting and mechanical properties of niobium may be affected. For example, while niobium is a conventional superconductor with $T_{\mathrm{c}}=9.25 \mathrm{~K}$, niobium hydrides are non-superconducting at $T>1.3 \mathrm{~K}$ and thus may have an unintended negative effect in important applications, such as superconducting radio frequency (SRF) cavities for particle accelerators, Josephson junctions, microresonators, or photon detectors.

SRF cavities are the key technology for all the particle accelerators for high-energy physics, nuclear physics, light sources, and accelerator-driven subcritical reactors. Cavities are made of bulk niobium with the residual resistivity ratio (RRR) of $\sim 300$ and hydrogen contamination is known to lead to the condition named "hydrogen $\mathrm{Q}$ disease" - a severe degradation of the cavity quality factor starting at very low accelerating fields ${ }^{1-4}$. The effect was attributed to the formation of non-superconducting niobium hydride phases, and it was quickly found that $\mathrm{Q}$ disease can be "cured" and prevented by vacuum annealing at $T>600^{\circ} \mathrm{C}$ for several hours, presumably due to the decrease of the bulk hydrogen concentration. More recently, smaller hydrides were proposed ${ }^{5,6}$ as a cause for another cavity limitation - a "high field Q slope", understanding of which is an area of active ongoing research.

Hydrogen can be absorbed by bulk niobium whenever natural oxide $\left(\mathrm{Nb}_{2} \mathrm{O}_{5}\right)$ passivating layer is absent on the surface. There is a number of such opportunities during cavity fabrication and processing. Known examples are buffered chemical $(\mathrm{BCP})$ or electropolishing $(\mathrm{EP})^{7}$, reabsorption from the furnace upon cooldown after 600$800^{\circ} \mathrm{C}$ baking $^{8-10}$, and centrifugal barrel polishing (CBP)

\footnotetext{
a) Electronic mail: Corresponding author: aroman@fnal.gov
}

in the water-based solution ${ }^{11}$.

It is therefore important to systematically investigate for cavity-grade niobium and surface treatments associated with cavities the details of how hydride form, their morphology, and effect of trapping centers. In our previous work ${ }^{12}$ we reported observation of surface relief left by the formation of hydrides in a temperature range 77 $150 \mathrm{~K}$, from which we estimated spatial dimensions of the hydrides. It had a disadvantage that all the measurements were taken at room temperature when hydrides had already disappeared and consequently it provided only a "lower bound" on the hydride precipitation. In this article we present systematic low-temperature studies performed by utilizing laser confocal and optical microscopy coupled with the cryogenic stage.

At room temperature hydrogen atoms in cavity-grade niobium are distributed on random tetrahedral interstitial sites and, due to the high mobility, behave like a gas. This solid solution is called $\alpha$-phase. The solubility limit of this phase is $\sim 4$ atomic percent of hydrogen at room temperature and is reduced significantly as temperature is decreased. At $100 \mathrm{~K}$ the maximum solubility is only several atomic ppm of hydrogen. When hydrogen concentration is above the solubility limit different stoichiometric and non-stoichiometric hydrides can form.

A variety of experimental methods has been applied in the past to study the $\mathrm{Nb}-\mathrm{H}$ system at different hydrogen concentrations: X-ray, neutron and electron diffraction, differential thermal analysis (DTA), resistivity, susceptibility, optical microscopy, and transmission (TEM) and scanning (SEM) electron microscopies. We briefly summarize the findings below.

Most intensively studied is the $\beta$-phase ${ }^{13-19}$. Neutron diffraction experiments ${ }^{20}$ showed that it is formed by ordering of the hydrogen interstitials on tetrahedral interstitial sites and has a face centered orthorhombic structure of host niobium atoms. The $\alpha-\beta$ solvus line was determined by using resistivity ${ }^{21-23}$, internal friction ${ }^{24}$ and DTA $^{25}$ methods. Since niobium itself has a cubic bodycentered crystal structure, the formation of $\beta$-phase is accompanied by about $11.9 \%$ molar volume increase ${ }^{26-28}$, 
leading to a deformation of the surrounding niobium ma$\operatorname{trix}^{29}$. This creates a lot of dislocations, especially at the interface between $\alpha$ and $\beta$ phases ${ }^{13}$.

$\beta$-phase inclusions can range in size from hundreds of nanometers ${ }^{15}$ to hundreds of microns ${ }^{13,30}$, and usually their size depends on the cooldown rate - the faster the cooldown the smaller the hydrides ${ }^{17}$. The inclusions have a tendency to be bounded by low index planes such as [100] or [110].

At high hydrogen concentrations (or at lower temperatures) other phases were observed - $\gamma$-phase ${ }^{16,18}, \delta$ phase $^{16,18,31}, \zeta$-phase ${ }^{14}$, and $\epsilon$-phase ${ }^{14,15}$.

Numerous studies ${ }^{13,16-19}$ showed the effectiveness of different optical techniques - hydrides were observed by bright field and dark field imaging, in polarized light and by using interference techniques. Of importance is a fact that the samples must be virtually free of deformation layers. However, transitions $\beta \rightarrow \zeta \rightarrow \epsilon$ are not observable by metallographic techniques since all these phases have identical structure of host niobium atoms and lattice parameters ${ }^{18}$.

Our presented studies rely on the optical contrast and morphological changes for detection of hydrides and are focused on the particular surface treatments of relevance to SRF cavities. Furthermore, 3D-profiles of hydrides, measured by the laser confocal scanning microscopy (LCSM), and an electron backscattered diffraction (EBSD) characterization of crystallographic orientations present the first measurements of this kind on niobium.

\section{EXPERIMENTAL METHODS}

We have used square samples of $\sim 3 \times 15 \times 15 \mathrm{~mm}$ cut by wire electron discharge machining (EDM) from fine grain (grain size $50 \mu \mathrm{m}$ ) and single grain $\mathrm{RRR} \sim 300$ niobium sheets from the same material batches as were used to manufacture 9-cell and 1-cell cavities with the state-ofthe-art performance.

Electropolishing (EP) and buffered chemical polishing (BCP) were performed using the in-house sample electropolishing setup with the standard acid solutions used for cavities. $800^{\circ} \mathrm{C}$ baking was done in the same vacuum furnace, which is used for cavities. $120^{\circ} \mathrm{C}$ baking was done using a continuously pumped vacuum tube with resistive heating on the outside walls. Tensile mechanical deformation of the samples was introduced using Instron machine.

For mechanical polishing samples were first embedded in either Bakelite or epoxy pucks and polished by the increasing grit size (120-600) sandpaper. It was followed by the automated polishing on the Textmet polishing cloth with $6 \mu \mathrm{m}$ size diamond solution for several hours. Final polishing was done on the vibrational polishing machine (vibromet) with the colloidal water-based $\mathrm{SiC}$ solution.

Interstitial hydrogen concentration in representative samples has been measured by vacuum extraction at ATI
TABLE I. Hydrogen concentration (atomic \%) after different treatments as measured by vacuum extraction.

\begin{tabular}{ccc}
\hline \hline Treatment & Single grain & Fine grain \\
\hline no treatment & $0.09 . .0 .11$ & $<0.03 . .0 .04$ \\
\hline $120 \mu \mathrm{m}$ EP & $0.10 . .0 .14$ & $<0.03$ \\
\hline $120 \mu \mathrm{m} \mathrm{BCP}$ & 0.11 & $<0.03$ \\
\hline $\mathrm{MP}$ & $\mathrm{N} / \mathrm{A}$ & $0.22-0.66$ depending on the duration \\
\hline \hline
\end{tabular}

Wah Chang using the LECO RH-404 hydrogen analyzer. In this method, a metal specimen, held by a single-use graphite crucible, is fused in an argon atmosphere using an impulse-type fusion furnace and hydrogen gas is detected by the thermistor bridge. Since complete sample melting occurs, the technique allows to measure the total content of hydrogen in the sample and is insensitive to ambiguities, which may arise in other techniques due to trapping of hydrogen by different kinds of lattice defects and grain boundaries.

For cryogenic measurements samples were glued with Apiezon $\mathrm{N}$ grease to the cold finger of the optical cryostat. Liquid helium -based gas flow cryostat allowed to reach temperatures down to about $5 \mathrm{~K}$. Real-time observations of niobium surface were done using a laser confocal optical microscope with a lateral resolution of the order of $1 \mu \mathrm{m}$ in a temperature range $5-300 \mathrm{~K}$. Lakeshore temperature controller was used to control and stabilize the temperature for observations.

To investigate the correlation between directions of hydrides growth and crystallographic directions we compared pictures of surface relief left by hydrides with EBSD patterns obtained by the use of scanning electron microscope.

\section{RESULTS}

Hydrogen concentration in some of the samples we used has been measured by the vacuum extraction technique at ATI Wah Chang with the results shown in Table I. The technique we used has a resolution of $\sim 0.03$ at.\% which makes it impossible to draw conclusions on the effect of treatments in the lower concentration range. As expected, mechanical polishing lead to the highest hydrogen concentration ranging from 0.22 to 0.66 at.\% depending on the polishing duration, whereas EP and $\mathrm{BCP}$ under conditions similar to cavities did not affect hydrogen content significantly. Interestingly, single grain samples had high hydrogen content of 0.09-0.11 at.\% even before any treatments, perhaps as a consequence of the method, by which the sheets are made from the large grain niobium ingot, which involves slicing ingot in water by wire electric discharge machine. 

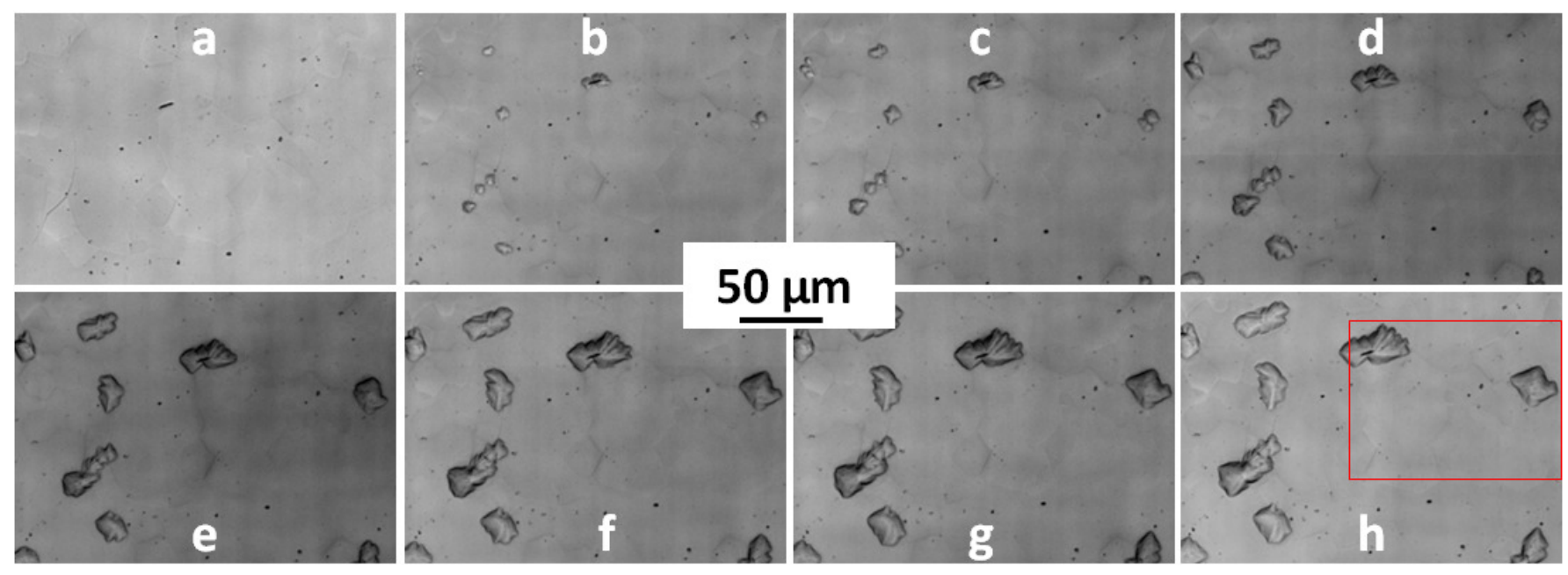

FIG. 1. Time evolution of hydrides observed on a mechanically polished fine grain sample with $C_{\mathrm{H}}=0.23$ at. $\%$ at $T=140 \mathrm{~K}$ : a) room temperature; b) after $60 \mathrm{~s}$ spent at $140 \mathrm{~K}$; c) $120 \mathrm{~s}$; d) $300 \mathrm{~s}$; e) $900 \mathrm{~s}$; f) $2700 \mathrm{~s}$; g) $6000 \mathrm{~s}$; h) $10800 \mathrm{~s}$.

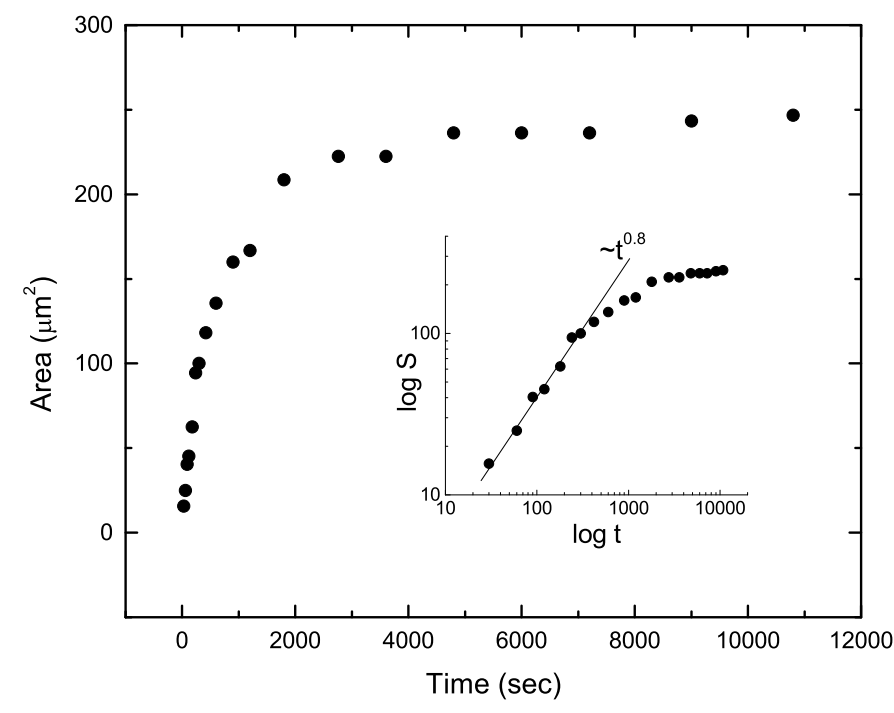

FIG. 2. Typical hydride surface area versus time for hydrides in Fig. 1

\section{A. Mechanically polished samples}

Among the samples we studied, mechanically polished samples showed the highest hydrogen concentration. Niobium SRF cavities with such a high hydrogen content, obtained for example after CBP, exhibit "hydrogen Q disease", and thus our cryogenic measurements allow to directly investigate the underlying microstructural cause of this effect. While cooling down, we continuously watched the surface of the samples in the laser confocal microscope and could immediately notice any morphological changes. Indeed, upon cool down from room temperature, clearly discernibly hydrides appeared at $T \sim 160 \mathrm{~K}$. Hydride precipitation could not be avoided even with the fastest cool down ( $\sim 10$ minutes $)$ to $5 \mathrm{~K}$ [Fig. 6a]. Thus MP samples provided an ideal system to investigate precipitation kinetics, morphology, effects of deformation, and of $120^{\circ} \mathrm{C}$ bake.

\section{Precipitation kinetics}

Samples were quickly cooled down to $140 \mathrm{~K}$ and kept at this temperature for 3 hours while images of the hydride growth were taken. Time evolution of patterns for the fine grain mechanically polished sample is presented in Fig. 1. In Fig. 2, typical surface area of one of the hydrides is plotted as a function of time spent at $140 \mathrm{~K}$. It shows that hydrides grow very fast during first $\sim 30$ minutes after which the process becomes much slower. But even after 3 hours of observation hydrides did not completely stop to grow.

After a 3 -hour hold at $140 \mathrm{~K}$ we rapidly $(\sim 20 \mathrm{~K} / \mathrm{min})$ cooled samples down to $5 \mathrm{~K}$. As soon as the temperature reached $\sim 100 \mathrm{~K}$ smaller in size hydrides appeared in the areas between the hydrides grown at $140 \mathrm{~K}$ [see Fig. 3]. These smaller hydrides are formed preferentially at the grain boundaries. The same finding was confirmed on two other fine grain mechanically polished samples.

\section{Hydride morphology}

Large hydrides, which formed first, had a characteristic shape for each particular grain orientation. Surface profiles of one of such hydrides obtained by LCSM at $160 \mathrm{~K}$ is shown in Fig. 4. All of the hydrides represented bumps on the surface with the characteristic height of about $3 \mu \mathrm{m}$. They appeared almost immediately and their height remained almost unchanged during 


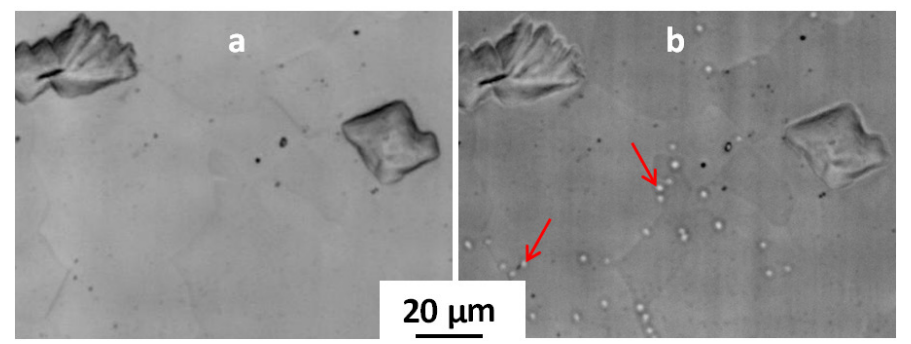

FIG. 3. Enlarged view of the area in Fig. 1h: a) after keeping the sample for 3 hours at $140 \mathrm{~K}$; b) after additional cooling to $100 \mathrm{~K}$. Arrows indicate small hydrides formed at $100 \mathrm{~K}$.
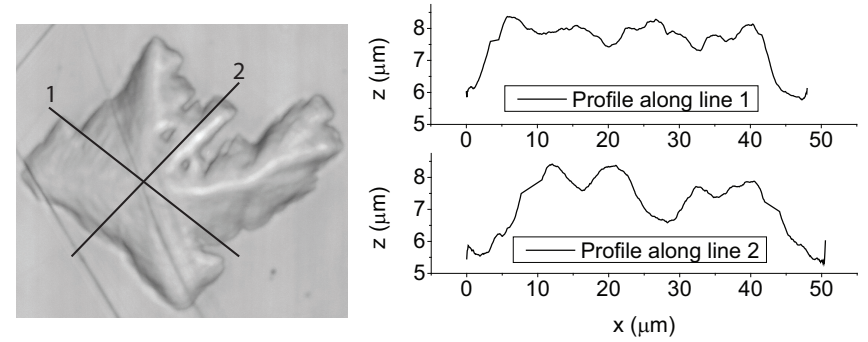

FIG. 4. Laser image of one of the hydrides with two surface profiles obtained along the labeled lines. Notice difference in $x$ and $z$ scales.

further growth that was proceeding essentially in the surface plane only. Observed heights are much larger than our previous "lower bound" estimate $(\sim 100 \mathrm{~nm})$ made from the observations of surface relief due to hydrides formation ${ }^{12}$.

Hydride shape appeared to be dependent upon the crystallographic orientation in each particular grain. This effect was especially pronounced in the case of a single grain material [see Fig. 10b, f]. To investigate it further we collected EBSD patterns around the hydrideinduced dents in the single grain sample. As can be seen from Fig. 5b, sharp borders of the dent appear to be close to the (110) family of crystallographic directions of the host niobium lattice.

\section{Effect of deformation}

To investigate if plastic deformation has any influence on the hydride formation we prepared fine grain samples with the varying degree of tensile deformation. All deformed samples were then mechanically polished in the same batch to ensure the same hydrogen pickup conditions. Vacuum extraction analysis after polishing showed that hydrogen concentration was the same $0.60 \pm 0.05$ at.\% in all the samples, thus the only difference was the degree of deformation. The results of fast cooldown tests on these samples are presented in Fig. 6. One can see a clear effect: higher deformation leads to a smaller number of hydrides. Interestingly, hydrides
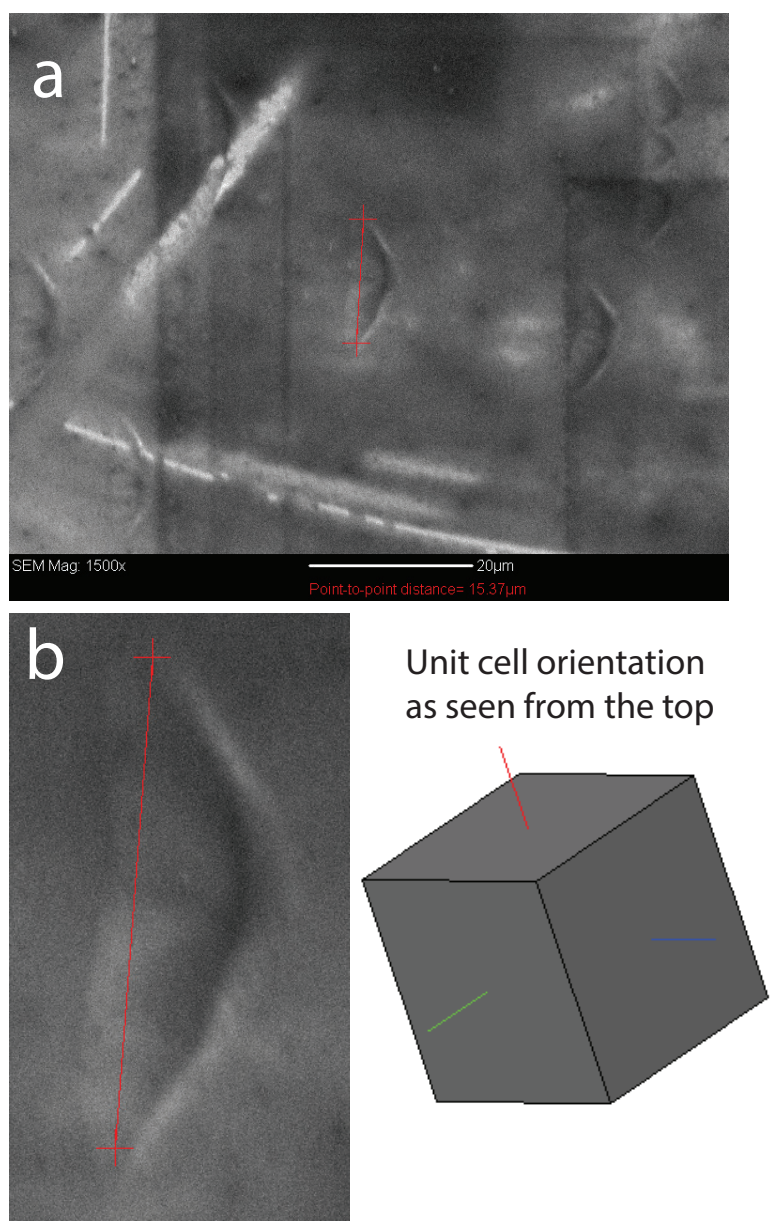

FIG. 5. a) SEM image of dents on the surface of the single grain mechanically polished sample after cryohold; b) zoomin of one of the dents with the corresponding crystallographic orientation of the surrounding niobium lattice measured by EBSD shown on the right.

also started to form at higher temperatures in deformed samples and temperatures of the first hydride observation were $T=160,170,180$ and $180 \mathrm{~K}$ respectively for the samples in Fig. 6.

We repeated the same experiment with electropolishing for $120 \mu \mathrm{m}$ material removal instead of mechanical polishing as a final step. In this case no hydrides were formed during cooldown [see Fig. 9h], likely due to the lack of significant hydrogen contamination by electropolishing as opposed to mechanical polishing in the first experiment.

\section{Second and subsequent cool downs}

We have found that hydride formation sites and kinetics were altered on the subsequent cooldowns as compared to the first one. This effect is shown in Fig. 7 where upon the second cooldown the hydrides started nucleating at a higher temperature (190 K vs. $160 \mathrm{~K}$ in the first 


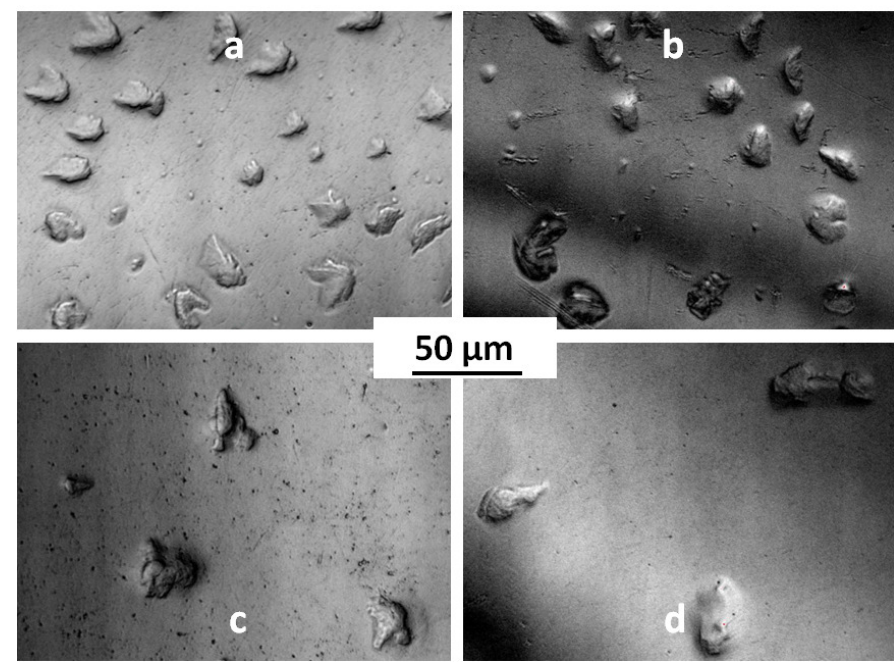

FIG. 6. Patterns obtained after fast cooldown to $5 \mathrm{~K}$ of mechanically polished fine grain samples with different degrees of prior mechanical deformation: a) no deformation, b) $7 \%$ tension, c) $10 \%$ tension, d) $12 \%$ tension

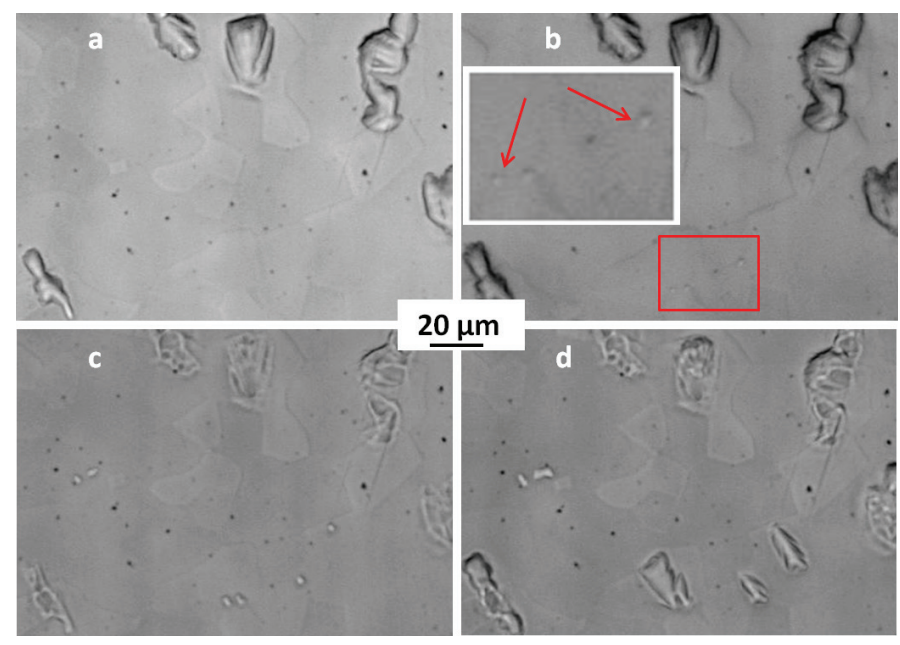

FIG. 7. Hydrides observed on the same sample during: a) 1st cooldown, 2 hours at $140 \mathrm{~K}$; b) additional cooling down to $90 \mathrm{~K}$, arrows indicate formation of small hydrides; c) 2nd cooldown - 140K; d) 2nd cooldown - after 2 hours at $140 \mathrm{~K}$.

cryocycle) and at different sites, which seem to correspond to the sites of the second phase formation at $90 \mathrm{~K}$ in the first cooldown. Additionally, localized hydride formation has been also observed within the "skeleton" left by the large hydrides on the first cooldown, although with the much smaller sizes. Upon the third cooldown the hydride formation was similar to the second.

\section{Effect of $120^{\circ} \mathrm{C}$ bake}

In order to investigate the effect of $120^{\circ} \mathrm{C}$ bake we split several mechanical samples in two halfs to preserve the same hydrogen content, and then baked one of the halfs at $120^{\circ} \mathrm{C}$ in vacuum for 48 hours. While nucleation and evolution of hydrides at $140 \mathrm{~K}$ was found to be virtually unchanged, the behavior after further cool down was found to be completely different - no small hydrides appeared upon cool down to $100 \mathrm{~K}$ and below as shown in Fig. 8 .

\section{B. Precipitation after standard treatments}

In this series of experiments we put samples through a sequence of standard cavity processing steps and performed cryogenic tests after each treatment. Treatments included bulk material removal of $100-200 \mu \mathrm{m}$ by EP or $\mathrm{BCP}, 800^{\circ} \mathrm{C}$ vacuum bake for 3 hours, "light" material removal of $20-40 \mu \mathrm{m}$, and a $120^{\circ} \mathrm{C}$ vacuum bake for 48 hours. Typically, it takes $\sim 45$ minutes for all parts of cavity to get to liquid helium temperatures. Of primary importance for hydride precipitation is the time spent in the temperature range between about 70 and $170 \mathrm{~K}$, and it is typically 5 to 15 minutes for what is usually referred to as a "fast cavity cooldown". Thus we tried to keep the same cool down rate in this series of experiments to "simulate" cavity conditions. As in previous experiments, we continuously monitored surface of the samples with the laser confocal optical microscope, which allowed to immediately notice any changes caused by the formation of hydrides.

Results of the cooldown tests for the fine grain samples are shown in Fig. 9. We have not observed any hydride formation after any of the studied treatments.

An analogous set of experiments was produced with single grain samples. The results are presented in Fig. 10. In this case we have observed the formation of hydrides in samples after bulk EP and BCP. Hydrides appeared at $140 \mathrm{~K}$ in the form of "lens-like" structures parallel to each other and grew along the longest dimension as temperature decreased down to $\sim 100 \mathrm{~K}$. With further temperature decrease both dimensions and a number of hydrides remained constant. Thermal hysteresis has been observed and upon warming up hydrides disappeared at a higher temperature of about 200 K. Surface relief (dents), which look similar to what we observed in our previous studies $^{12}$, remained after hydrides disappeared. After samples were baked at $800^{\circ} \mathrm{C}$ for 3 hours, no observable hydrides could be found during cooldown, and also none were found after any of the subsequent treatments, which included $40 \mu \mathrm{m} \mathrm{EP}$ and $120^{\circ} \mathrm{C}$ baking for 48 hours.

A summary of the fast cooldown test results is presented in Table II. A clear difference is apparent between the precipitation of hydrides in single grain and fine grain samples. Vacuum gas extraction measurements [Table I] suggest that this difference is most likely caused by the difference in hydrogen concentration in single and fine grain niobium even before any treatments are applied. Thus it seems that the only treatment, which has led to the strong hydrogen contamination manifested by the 

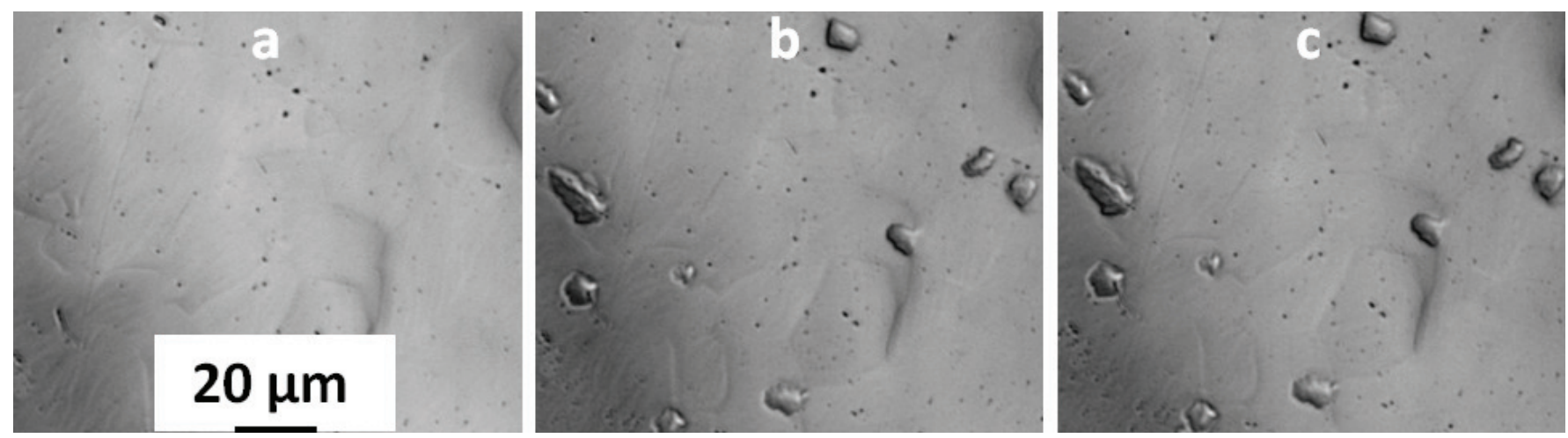

FIG. 8. Hydrides observed on a mechanically polished sample after additional $120^{\circ} \mathrm{C}$ baking for 48 hours: a) room temperature; b) after $140 \mathrm{~K}, 2$ hours; c) after further cooling down to $5 \mathrm{~K}$. Notice an absence of the second phase precipitation unlike the unbaked sample.
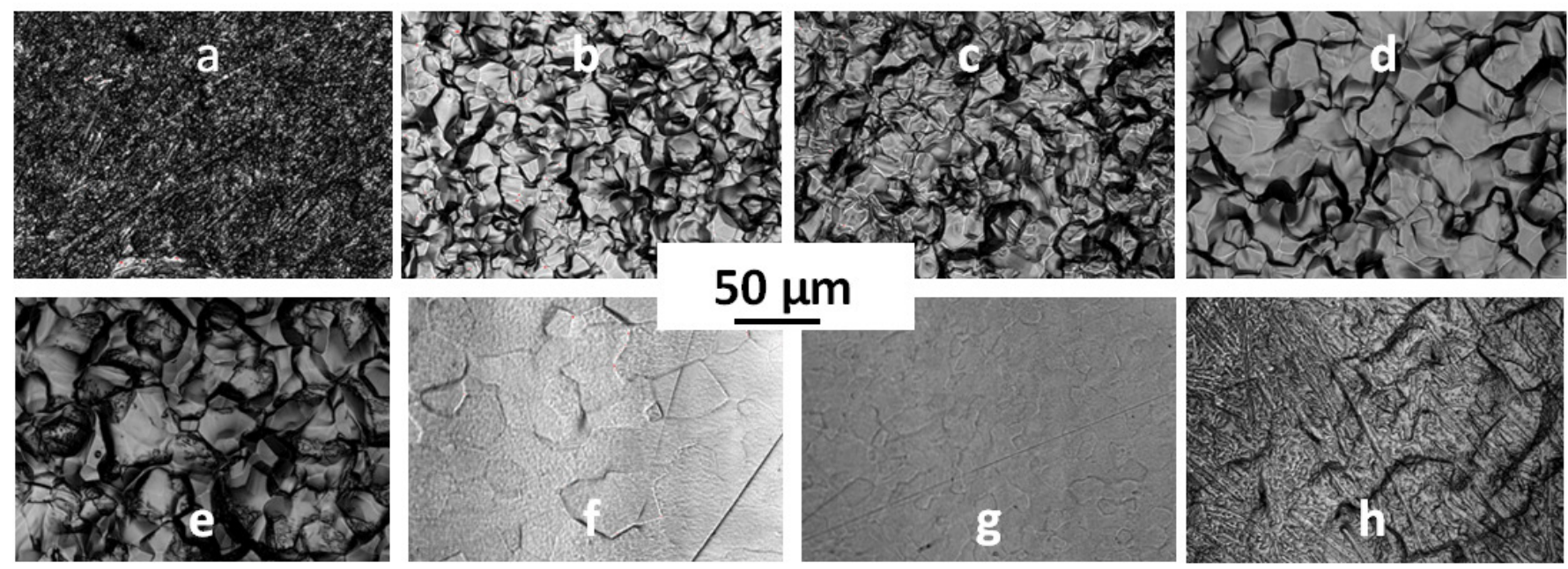

FIG. 9. Optical microscope patterns obtained after fast cooldown to $5 \mathrm{~K}$ of fine grain samples after different treatments: a) "as is" sample cut from niobium sheet; b) $120 \mu \mathrm{m}$ BCP material removal; c) $120 \mu \mathrm{m}$ BCP material removal $+800^{\circ} \mathrm{C}$ baking for 3 hours; d) $120 \mu \mathrm{m} \mathrm{BCP} \mathrm{material} \mathrm{removal}+800^{\circ} \mathrm{C}$ baking for 3 hours $+40 \mu \mathrm{m} \mathrm{BCP}$ material removal; e) $120 \mu \mathrm{m}$ BCP material removal $+800^{\circ} \mathrm{C}$ baking for 3 hours $+40 \mu \mathrm{m} \mathrm{BCP}$ material removal $+120^{\circ} \mathrm{C}$ baking for 24 hours; f) $120 \mu \mathrm{m} \mathrm{EP}$ material removal; g) $120 \mu \mathrm{m}$ EP material removal $+800^{\circ} \mathrm{C}$ baking for 3 hours; h) 10 per cent tension $+120 \mu \mathrm{m}$ EP material removal

formation of large hydrides at cryogenic temperatures is mechanical polishing.

\section{DISCUSSION}

Our investigations show that niobium hydrides can be observed by laser and optical microscopy coupled with the cryogenic sample stage, and this technique allows us to draw multiple conclusions on the hydride-related effects in SRF niobium cavities.

As described in the literature, niobium hydrides are non-superconducting at $T=2 \mathrm{~K}$, and, therefore, should lead to the strong microwave dissipation in SRF cavities. A strong correlation between the presence or absence of "Q disease" in cavities and the presence or absence of hydrides in similarly treated samples suggests that large
TABLE II. Fast cooldown test results after different treatments. Shown are the temperatures of hydrides appearance during cooldown and disappearence during warmup; "-" indicates an absence of hydrides.

\begin{tabular}{ccc}
\hline \hline Treatment & Single grain & Fine grain \\
\hline no treatment & - & - \\
\hline $120 \mu \mathrm{m} \mathrm{EP}$ & $140 \mathrm{~K} / 200 \mathrm{~K}$ & - \\
$+800^{\circ} \mathrm{C} 3 \mathrm{hrs}$ & - & - \\
$+40 \mu \mathrm{m} \mathrm{EP}$ & - & - \\
$+120^{\circ} \mathrm{C} 24 \mathrm{hrs}$ & - & - \\
\hline $120 \mu \mathrm{m} \mathrm{BCP}$ & $140 \mathrm{~K} / 200 \mathrm{~K}$ & - \\
$+800^{\circ} \mathrm{C} 3 \mathrm{hrs}$ & - & - \\
$+40 \mu \mathrm{m} \mathrm{BCP}$ & - & - \\
$+120^{\circ} \mathrm{C} 24 \mathrm{hrs}$ & - & - \\
\hline $\mathrm{MP}$ & $\mathrm{N} / \mathrm{A}$ & $160 \mathrm{~K} / 200 \mathrm{~K}$ \\
$+800^{\circ} \mathrm{C} 3 \mathrm{hrs}$ & - & - \\
\hline \hline
\end{tabular}



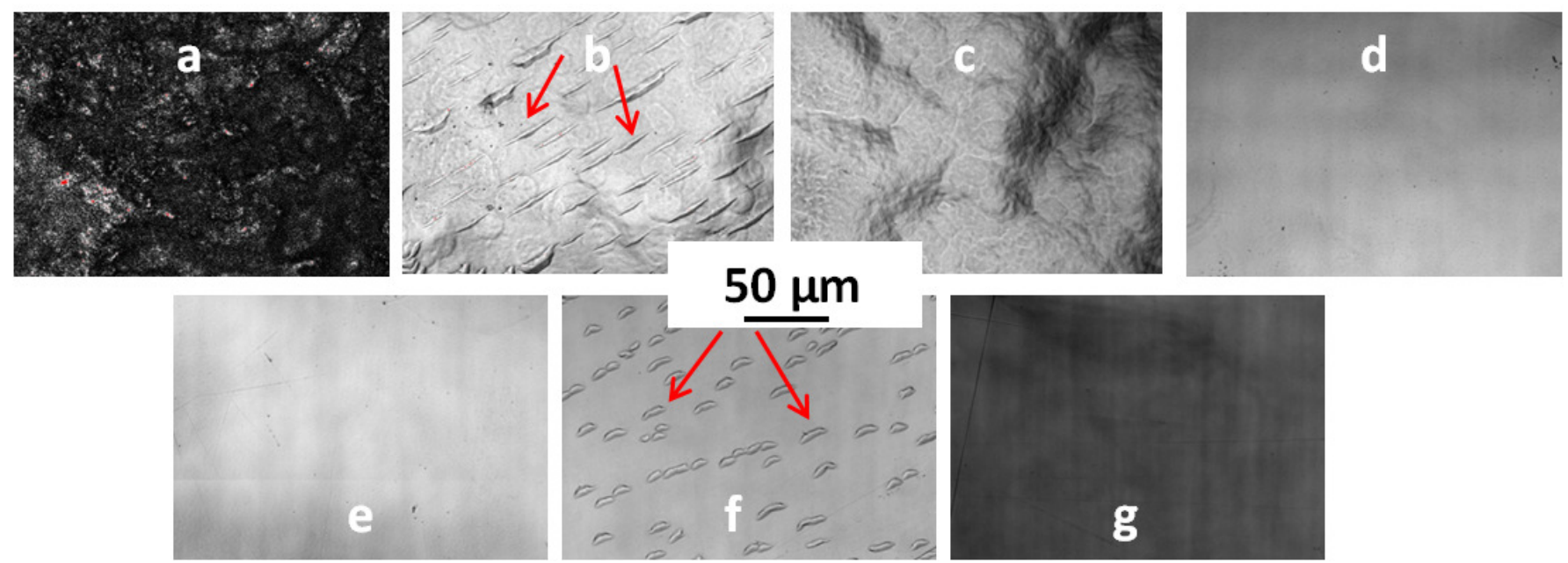

FIG. 10. Patterns obtained after fast cooldown to $5 \mathrm{~K}$ of single grain samples after different treatments: a) "as is" sample cut from niobium sheet; b) $120 \mu \mathrm{m}$ EP material removal; c) $120 \mu \mathrm{m}$ EP material removal $+800^{\circ} \mathrm{C}$ baking for 3 hours; d) $120 \mu \mathrm{m}$ $\mathrm{EP}$ material removal $+800^{\circ} \mathrm{C}$ baking for 3 hours $+40 \mu \mathrm{m} \mathrm{EP}$ material removal; e) $120 \mu \mathrm{m} \mathrm{EP}$ material removal $+800^{\circ} \mathrm{C}$ baking for 3 hours $+40 \mu \mathrm{m}$ EP material removal $+120^{\circ} \mathrm{C}$ baking for 24 hours; f) $120 \mu \mathrm{m} \mathrm{BCP}$ material removal; g) $120 \mu \mathrm{m}$ $\mathrm{BCP}$ material removal $+800^{\circ} \mathrm{C}$ baking for 3 hours. Arrows indicate hydrides formed during cooldown.

hydrides we observe are actually the ones responsible for the "Q disease".

We have investigated if different treatments used in cavity productions lead to the formation of hydrides during cooldown to cavity operational temperatures of around $2 \mathrm{~K}$. Among the standard treatments (EP, BCP, $\mathrm{CBP}$ ), only mechanical polishing (analogous to CBP) has led to the observable large hydrides in fine grain samples. This is caused by the strong hydrogen loading as detected by the vacuum gas extraction, and is in agreement with the cavity evidence showing that $800^{\circ} \mathrm{C}$ degassing is necessary for centrifugal barrel polished (tumbled) cavities ${ }^{11}$, which are otherwise limited by a severe " $Q$ disease". Lack of hydrides after other treatments may explain the fact that some cavities after EP or BCP have no Q-disease even without $800^{\circ} \mathrm{C}$ baking.

In single grain samples hydrides were found after all material removal treatments (EP, BCP, mechanical polishing) unless $800^{\circ} \mathrm{C}$ was performed. However, for EP and BCP it is likely not caused by the treatments, but is a consequence of the high starting concentration of hydrogen in a single grain niobium sheet. This high hydrogen content may be due to the sheet slicing method (wire electric discharge machine), and indicates that hydrogen degassing may be required for large and single crystal niobium sheets. It is interesting in this respect that we could not observe any hydrides on the surface of single grain samples in as-received condition. However, the as-received single grain sheet was very rough and had a very thick oxide layer. This surface condition limited the imaging quality, and also could lead to many trapping centers on the surface suppressing the hydride formation.

According to our results, whenever niobium is contaminated with hydrogen, $800^{\circ} \mathrm{C}$ baking for 3 hours is an effective way to reduce the bulk hydrogen concentration and to preclude the formation of hydrides. This is in line with a well known fact that $800^{\circ} \mathrm{C}$ baking removes the $\mathrm{Q}$ disease $^{2-4}$.

Whether hydrides responsible for the Q disease are islands or a layer remained an open question for almost two decades ${ }^{32}$. Our data shows that hydrides have a shape of islands, which punch out of the surface. Inplane dimensions depend on the concentration and the time spent in the precipitation temperature range. After several hours at 140-160 K typical heights of hydrides were about $3 \mu \mathrm{m}$, which is much larger than the depth of the surface relief left by hydrides dissociation reported in $^{12}$, where dents had a characteristic height of $150 \mathrm{~nm}$. We believe the reason is that surface relief is "proportional" to the hydride-induced deformation or in other words to the difference in volumes of hydride and niobium phases, and this difference is much smaller than the volume of the hydride phase.

Fig. 2 demonstrates that it takes only $\sim 30$ minutes for the hydrides to reach almost maximal size. Since the time spent in the precipitation range for typical "fast" cavity cooldowns is of the same order, it means that even "fast cooldown" will not help to avoid hydride formation when hydrogen concentration is high enough. It also means that for thin hydrogen-reach layers found even after $800^{\circ} \mathrm{C}$ vacuum heat treatments there is always enough time for hydride formation. While we have not observed any hydrides after $800^{\circ} \mathrm{C}$ annealing in any of our samples, we are limited in spatial resolution, and we can only conclude that hydrogen concentration is not enough to form observable micrometer-size hydrides.

Hydride growth kinetics may help addressing several questions regarding hydrogen distribution in the sample and spatial localization of hydride precipitation. We can 


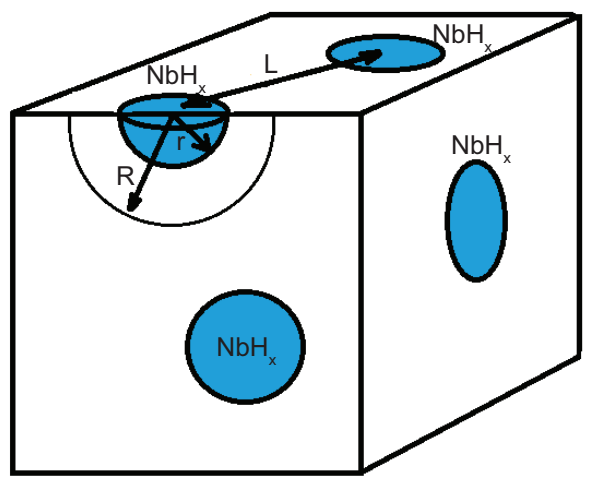

FIG. 11. Schematic of the hydride precipitation model.

describe the hydride growth process by a simple diffusion model where the growth is controlled by hydrogen atoms diffusing towards nucleation centers. The discussion of what defects can serve as nucleation centers is still open but we think that vacancies and hydrogen-vacancies complexes are among likely candidates ${ }^{5,12}$. We start with the assumption that hydrogen is uniformly distributed in the sample volume, and hydride nuclei are spaced by the distance $L$. The schematic used for this model is shown in Fig. 11.

Then the number $N(t)$ of hydrogen atoms reaching each surface hydride $\left(\mathrm{NbH}_{\mathrm{x}}\right)$ in the process of diffusion by time $t$ is given by

$$
N(t)=\frac{2}{3} \pi R(t)^{3} n_{\mathrm{H}}^{\mathrm{bulk}}
$$

where $R(t)=\sqrt{6 D t}$ is the diffusion radius, and $n_{\mathrm{H}}^{\text {bulk }}$ is the bulk hydrogen concentration. On the other hand, the number of hydrogen atoms in the hydride equals $V n_{\mathrm{H}}^{\text {hydride }}$ where $V=\frac{2}{3} \pi r^{3}$ is the hydride volume, and $n_{\mathrm{H}}^{\text {hydride }}$ is the concentration of hydrogen in the hydride phase. Assuming that all hydrogen atoms reaching the hydride are converted into hydride phase we can write:

$$
\frac{2}{3} \pi R(t)^{3} n_{\mathrm{H}}^{\text {bulk }}=\frac{2}{3} \pi r(t)^{3} n_{\mathrm{H}}^{\text {hydride }}
$$

and hence

$$
r(t)=R(t)\left(\frac{n_{\mathrm{H}}^{\text {bulk }}}{n_{\mathrm{H}}^{\text {hydride }}}\right)^{1 / 3}=\sqrt{6 D t}\left(\frac{n_{\mathrm{H}}^{\text {bulk }}}{n_{\mathrm{H}}^{\text {hydride }}}\right)^{1 / 3}
$$

The area of the hydride should increase with time as $\pi r^{2} \propto t$. The growth process drastically slows down when diffusion volumes from adjacent hydrides begin to overlap and eventually ceases when free hydrogen concentration drops below the solubility limit. This is in good qualitative agreement with our observations of the hydride surface area growth shown in Fig. 2 where the area increases initially $\propto t^{0.8}$. The slightly slower process than predicted $(\propto t)$ can be explained by the decrease in hydrogen concentration $n_{\mathrm{H}}^{\text {bulk }}$ with time, which is neglected in our model.
While we cannot determine the exact hydride stoichiometry $\mathrm{NbH}_{\mathrm{x}}$ from our observations, for estimates we can safely assume that $x \sim 1$. In this case niobium concentration in the hydride $n_{\mathrm{Nb}}^{\text {hydride }}=n_{\mathrm{H}}^{\text {hydride }}$, and if we make a reasonable assumption that $n_{\mathrm{Nb}}^{\text {hydride }}=n_{\mathrm{Nb}}^{\text {bulk }}$ then we can use $n_{\mathrm{H}}^{\text {bulk }} / n_{\mathrm{Nb}}^{\text {bulk }}$ known from vacuum extraction measurements instead of the ratio $n_{\mathrm{H}}^{\text {bulk }} / n_{\mathrm{H}}^{\text {hydride }}$. As a consistency check for the model we can estimate the value of the diffusion constant $D$ based on the hydride surface area data in Fig. 2. From vacuum extraction $n_{\mathrm{H}}^{\text {bulk }} / n_{\mathrm{Nb}}^{\text {bulk }}=0.19$ at.\%, and using Eq.(3): $D \approx 1.4 \times 10^{-9} \mathrm{~cm}^{2} / \mathrm{s}$, which is in a reasonable agreement with literature ${ }^{33}$.

From the drastic slowdown of the hydride growth when $R(t)$ reaches the value of the order of the interhydride distance $L$ we can conclude that hydride precipitation should be happening throughout the sample volume since otherwise the supply from the bulk would not cease and the faster growth would continue. This conclusion is further supported by the estimates based on the total number of hydrogen atoms. For example, taking the surface area fraction of hydrides of about $\alpha=1.5 \%$ from Fig. 1h, an approximate height of $d_{\mathrm{H}}=3 \mu \mathrm{m}$ from the measurements in Fig. 4, and sample thickness $d=3 \mathrm{~mm}$, hydrogen atoms in the surface hydrides on two surfaces - if spread out over the whole sample volume - would give an atomic concentration of about $2 \cdot \alpha \cdot d_{\mathrm{H}} / d \approx 0.003$ at. $\%$. The factor 2 comes from the two main surfaces at which hydrides form (side surfaces are neglected). However, vacuum extraction shows that the concentration is much larger - 0.11 at.\%. If on the other hand the volume precipitation is assumed then the concentration is about $\alpha \cdot d_{\mathrm{H}} / L=1.5 \% \times 3 \mathrm{~mm} \times 50 \mu \mathrm{m} \approx 0.09$ at. $\%$ in an excellent agreement with vacuum extraction.

We attribute the formation of smaller hydrides at $100 \mathrm{~K}$ (after 3 hours hold at $140 \mathrm{~K}$ ) to the precipitation of another hydride phase, which has a lower solubility limit than the phase formed at $140 \mathrm{~K}$. This second phase is much smaller, likely due to the low free hydrogen concentration at $100 \mathrm{~K}$. It is unlikely that $120^{\circ} \mathrm{C}$ baking changes hydrogen concentration, which is also supported by previous studies ${ }^{34}$. It is also supported by our $140 \mathrm{~K} \mathrm{ob-}$ servations - there is no difference between baked and unbaked samples. Yet, small hydrides at $100 \mathrm{~K}$ are formed only on unbaked samples, and thus $120^{\circ} \mathrm{C}$ baking suppresses the formation of the second phase. We speculate that this lower temperature phase may be responsible for the high field $Q$ slope as suggested by the proximitycoupled hydride model ${ }^{5}$. There are several possible scenarios for the suppression, which we would like to mention. In the first one the vacancies introduced by the $120^{\circ} \mathrm{C}$ bake trap some hydrogen, as was recently found in baked cavity cutouts ${ }^{6}$. In the second one, $120^{\circ} \mathrm{C}$ baking leads to the removal of the hydride nucleation centers. The third scenario may involve diffusion of oxygen from the partially dissociating niobium oxide ${ }^{35}$.

The difference between the first and subsequent cooldowns and the effect of deformation may both be ex- 
plained by the hydrogen-dislocation interaction. Strong hydrogen attraction to dislocations, especially of edge character, is known to be responsible for the formation of Cottrell clouds with higher hydrogen concentration around dislocation cores in niobium ${ }^{36,37}$.

It is known that strong plastic deformation at hydride formation sites produces large dislocation densities ${ }^{13,29}$. This leads to the formation of Cottrell clouds, less uniform local hydrogen concentration after the first cryocycle, and a smaller amount of free hydrogen. Higher concentrations around the dislocations then may explain the observed higher temperatures of the hydride formation upon the subsequent cool downs. It is not possible to distinguish by laser observations if the hydride phase forming upon subsequent cryocycles is the same as the "high" temperature or "low" temperature phase in the first cool down. This question may be clarified by future nanostructural studies.

For the samples with different degrees of tensile deformation (and the same hydrogen concentration) the surface density of hydrides observed during cooldown varies significantly in a systematic way - higher deformation leads to the lower observable hydride density. In this case dislocations are created more uniformly throughout the volume (as opposed to localized areas in the case of hydride-induced), and thus a possible scenario is that much higher volume density of Cottrell clouds leads to much smaller dispersed hydrides, which are therefore unobservable in our setup. The amount of free hydrogen available to form larger (observable) hydrides is then reduced leading to the lower observed density. It is in a certain way an analogy to the fact that cavities made of reactor grade niobium do not suffer from the $Q$ disease $^{38}$, an effect attributed to the reduced amount of free hydrogen due to trapping by interstitial impurities.

\section{CONCLUSIONS}

We have developed a technique for direct observation of the formation and evolution of niobium hydrides on the surface of cavity-grade niobium, and have investigated effects of different treatments used on SRF niobium cavities. Our findings implicate large island-shaped hydrides in the emergence of the $Q$ disease, consistent with the prevailing model. Growth of hydrides is reasonably described by a 3D diffusion model. Binding hydrogen by defects, which leads to the suppression of hydride precipitation, has been directly demonstrated in controlled deformation experiments. A strong effect of the $120^{\circ} \mathrm{C}$ bake on the lower temperature hydride phase precipitation has been found.

\section{ACKNOWLEDGMENTS}

We are thankful to M. Bossert, R. Schuessler, and D. Hicks for their help with sample preparation, to S.
Shishkin for the help with digital processing of the images, and to H. Padamsee for useful discussions. This work was partially funded by the DOE Office of Nuclear Physics. Fermilab is operated by Fermi Research Alliance, LLC under Contract No. DE-AC02-07CH11359 with the United States Department of Energy.

${ }^{1}$ S. Isagawa, J. Appl. Phys., 51, 6010 (1980).

${ }^{2}$ B. Aune, B. Bonin, J. M. Cavedon, M. Juillard, A. Godin, C. Henriot, P. Leconte, H. Safa, A. Veyssiere, and C. Zylberajch, in Proceedings of the 1990 LINAC Conference (1990) pp. 253-255.

${ }^{3} \mathrm{~B}$. Bonin and R. W. Roth, in Proceedings of the 5th Workshop on RF Superconductivity (1991) pp. 210-244.

${ }^{4}$ C. Z. Antoine, B. Aune, B. Bonin, J. Cavedon, M. Juillard, A. Godin, C. Henriot, P. Leconte, H. Safa, A. Veyssiere, A. Chevarier, and B. Roux, in Proceedings of the Fifth Workshop on RF Superconductivity, DESY, Hamburg, Germany (1991) pp. 616-634.

${ }^{5}$ A. Romanenko, F. Barkov, L. D. Cooley, and A. Grassellino, Supercond. Sci. Tech., 26, 035003 (2013).

${ }^{6}$ A. Romanenko, C. J. Edwardson, P. G. Coleman, and P. J. Simpson, Appl. Phys. Lett., 102, 232601 (2013).

${ }^{7}$ T. Higuchi, K. Saito, Y. Yamazaki, T. Ikeda, and S. Ohgushi, in Proceedings of the 10th Workshop on RF Superconductivity (2001) pp. 427-430.

${ }^{8}$ K. Faber and H. Schultze, Scripta Metallurgica, 6, 1065 (1972). ${ }^{9}$ S. Isagawa, J. Appl. Phys., 51, 4460 (1980).

${ }^{10}$ A. Grassellino, A. Romanenko, A. Rowe, L. D. Cooley, C. Ginsburg, and V. P. Yakovlev, in Proceedings of IPAC'12, WEPPC115 (2012).

${ }^{11} \mathrm{~T}$. Higuchi and K. Saito, in Proceedings of the 11th Workshop on RF Superconductivity (2003) pp. 572-578.

${ }^{12}$ F. Barkov, A. Romanenko, and A. Grassellino, Phys. Rev. ST Accel. Beams, 15, 122001 (2012).

${ }^{13}$ H. K. Birnbaum, M. L. Grossbeck, and M. Amano, J. Less. Comm. Metals, 49, 357 (1976).

${ }^{14}$ T. Schober, Phys. Stat. Sol., 30, 107 (1975).

${ }^{15}$ T. Schober, Scripta Metallurgica, 7, 1119 (1973).

${ }^{16} \mathrm{~T}$. Schober and U. Linke, Metallography, 9, 309 (1976).

${ }^{17}$ T. Schober and U. Linke, J. Less-Common Met., 44, 63 (1976).

${ }^{18}$ T. Schober and U. Linke, J. Less-Common Met., 44, 77 (1976).

${ }^{19}$ J.-M. Welter and F. J. Johnen, Z. Phys. B, 27, 227 (1977).

${ }^{20}$ V. Somenkov, A. Gurskaya, M. Zemlyanov, M. Kost, N. Chernoplekov, and A. Chertkov, Sov. Phys.-Solid St., 10, 1076 (1968).

${ }^{21}$ D. Westlake, Trans. AIME, 245, 287 (1969).

${ }^{22}$ G. Pfeiffer and H. Wipf, J. Phys. F, 6, 167 (1976).

${ }^{23}$ J. P. Welter, M. A. Pick, T. Schober, J. Hauck, H. F. Fenzl, and H. Wenzl, in Proceedings of the 2nd International Congress (1977).

${ }^{24}$ O. Buck, D. Thompson, and C. Wert, J. Phys. Chem. Solids, 32, 2331 (1971).

${ }^{25} \mathrm{H}$. Wenzl and J.-M. Welter, "Current topics in materials science," (North-Holland Publishing Company, 1978).

${ }^{26}$ M. Pick, in Int. Meeting Hydrogen in Metals, Vol. 1 (1972) pp. 90-93.

${ }^{27}$ W. Albrecht, M. Mallett, and W. Goode, J. Electrochem. Soc., 105, 219 (1958).

${ }^{28}$ C. Wainwright, A. Cook, and B. Hopkins, J. Less-Common Met., 6, $362(1964)$.

${ }^{29}$ M. Amano and Y. Sasaki, Phys. Stat. Sol. A, 19, 405 (1973).

${ }^{30}$ H. Paxton, J. Sheehan, and W. Babyak, Trans. AIME, 725 (215).

${ }^{31}$ T. Schober, Phys. Stat. Sol. A, 29, 395 (1975).

${ }^{32} \mathrm{R}$. W. Roth, in Proceedings of the 6th Workshop on RF Superconductivity (1993).

${ }^{33}$ C. Baker and H. K. Birnbaum, Acta Metall., 21, 865 (1973).

${ }^{34}$ A. Romanenko and L. V. Goncharova, Supercond. Sci. Tech., 24, 105017 (2011). 
${ }^{35}$ C. Benvenuti, S. Calatroni, and V. Ruzinov, in Proceedings of the 10th Workshop on RF Superconductivity (Tsukuba, Japan, 2001) p. 441.
${ }^{36}$ J. A. Rodriguez and R. Kirchheim, Scripta Metallurgica, 17, 159 (1983).

${ }^{37}$ E. Krautz, J. Refr. Met. and Hard Mat., 12, 315 (1993).

${ }^{38} \mathrm{~K}$. Saito and P. Kneisel, in Proceedings of the 5th Workshop on RF Superconductivity (DESY, Hamburg, Germany, 1991) pp. 665-679. 